JOURNAL OF THEORETICAL

AND APPLIED MECHANICS

55, 3, pp. 1101-1108, Warsaw 2017

DOI: $10.15632 /$ jtam-pl.55.3.1101

\title{
UBIQUITIFORMAL FRACTURE ENERGY
}

\author{
Zhuo-Cheng Ou, Min Yang, Guan-Ying Li, Zhuo-Ping Duan, Feng-Lei Huang \\ State Key Laboratory of Explosion Science and Technology, School of Mechatronics, Beijing Institute of Technology, Beijing, \\ China; e-mail: zcou@bit.edu.cn
}

\begin{abstract}
The ubiquitiformal fracture energy is proposed in the paper and its explicit expression is obtained. Moreover, the numerical results for concrete are found to be in good agreement with those for the critical strain energy release rate. The discrepancy between the numerical results of the traditional fracture energy and the critical strain energy release rate can be explained reasonably, which implies that the ubiquitiformal fracture energy should be taken as an available fracture parameter of materials. Finally, it is numerically found for some concrete that there is not size effect for the ubiquitiformal fracture energy.
\end{abstract}

Keywords: fractal, ubiquitiform, fracture energy, size effect

\section{Introduction}

As is well known, pioneered by the work of Mandelbrot et al. (1984), the fractality of fracture surfaces in various kinds of materials such as concrete (Saouma et al., 1990; Saouma and Barton, 1994), steel (Mandelbrot et al., 1984; Underwood, 1986), ceramic (Mecholsky, 1989) and rock (Krohn and Thompson, 1986; Radlinski et al., 1999) has been verified experimentally, which has gradually lead to the establishment of the emergent fractal fracture mechanics over the past three decades. Naturally, it is a reasonable desire that some important physical concepts or parameters in the classical fracture mechanics can be extended directly into the fractal one but, unfortunately, this is not the case sometimes. For example, fracture energy or, more scholarly, the strain energy release rate, is one of the significant properties characterizing the fracture property of materials in the classical fracture mechanics and defined as the energy required to create a unit new crack surface (in integral dimension of $D=2$ ). However, it seems that there exists an intrinsical difficulty for extending such a traditional concept in the classical fracture mechanics into the fractal fracture mechanics, because of singularity of the integral dimensional measure or the immeasurability of the corresponding fractal such as the so-called fractal fracture energy. That is to say, the integral dimensional measures or, intuitively, the area of all the fractal fracture surfaces tend in general to infinity, which makes all the traditional fracture energy vanishing. In fact, over the past decades, to overcome such a difficulty and well describe fractal characteristics of a fractal crack as a direct extension of the concept of traditional fracture energy, some new density kinds of fractal fracture energy parameters defined on a unit fractal measure were proposed, such as the specific energy-absorbing capacity of unit fractal measure (Borodich, 1992, 1997, 1999), fractal fracture energy (Bažant, 1995, 1997a,b) as well as the renormalized fractal fracture energy (Carpinteri, 1994; Carpinteri and Ferro, 1994; Carpinteri et al., 2002), which have been used widely in practical applications. However, as was pointed out recently by $\mathrm{Ou}$ et al. (2014), such a concept of the fractal fracture energy seems now to be a little questionable, because these fractal fracture energies are both difficult to be determined in practice and lack unambiguous physical meanings (Bažant and Yavari, 2005). More importantly, such defined fractal fracture parameters are not appropriate to be taken as a measure of strength or toughness of materials. On account of that the comparison between the 
measures of two objects in different dimensions is radically meaningless, while the traditional fracture energy is indeed an important characteristic parameter of materials. For example, one can say that the material with a higher fracture energy has higher a load bearing capacity. Addison (2000) tried to deal with such an issue for fractal cracks by using the concept of socalled pre-fractal fracture surfaces. With the aid of a new-defined hypervolume, Addison (2000) obtained the ratio of the area of the pre-fractal fracture surface to the original smooth crosssectional area of the specimens. Taking the ratio as a modified factor, the pre-fractal fracture energy was obtained but, certainly, the fractal fracture energy was still divergent and hence could not be determined. Moreover, it was also found by Addison (2000) that the values of the pre-fractal fracture energy were remarkably coincident with the critical strain energy release rate determined by the fracture toughness relation, in which the fracture toughness and the elastic modulus were determined experimentally (Swartz and Kan, 1992). Although, as concluded by Addison (2000), the pre-fractal fracture energy can be a true material constant, it should be noticed that the formulation of the pre-fractal fracture energy under the concept of fractals is a little miscellaneous and the hypervolume of a fractal object seems to have no physical significance, and then becomes unnecessary.

As above mentioned, there are some intrinsical difficulties in the practical engineering applications of fractals, especially when the measure of the considered object must be taken into account. As was pointed out further by Ou et al. (2014), the fractal approximation of a physical object in nature is unreasonable because of divergence of the integral dimensional measure of the fractal. Moreover, to cover the shortage in fractal applications, a new concept of a ubiquitiform was proposed by $\mathrm{Ou}$ et al. (2014). It is believed that a real physical or geometrical object in nature should be ubiquitiformal rather than fractal. According to Ou et al. (2014), a ubiquitiform can be defined as a finite order self-similar (or self-affine) physical configuration constructed usually by a finite iterative procedure and, moreover, under the concept of the ubiquitiform, the singularity of the integral dimensional measure or the immeasurability of the fractal disappears.

In this paper, therefore, the fracture energy and its size effects are re-analyzed based on the concept of the ubiquitiform. A ubiquitiformal fracture energy is proposed and its explicit expression is obtained. Subsequently, the calculated numerical results of the ubiquitiformal fracture energy for concrete are compared with those for the critical strain energy release rate calculated by using the well-known fracture toughness relation. Furthermore, a similar size effect of the fracture energy to that derived by fractal theory is also obtained. This article is divided into four sections. After this brief introduction, the ubiquitiformal fracture energy and the size effect of the fracture energy are presented in Section 2. In Section 3, numerical results for the ubiquitiformal fracture energy are presented together with a brief discussion and, finally, some conclusions are drawn out in Section 4.

\section{Ubiquitiformal fracture energy of concrete}

In the classical fracture mechanics, the fracture energy $G$ is defined as the released energy $W$ divided by the opened fracture area $A$, namely

$$
G=\frac{W}{A}
$$

Thus, for a specimen with a smooth square cross-section of side length $l, A=l^{2}$, the traditional fracture energy is

$$
G=\frac{W}{l^{2}}
$$


On the other hand, when taking the same cross-section as a ubiquitiformal surface, the ubiquitiformal area $A_{u f}$ is

$$
A_{u f}=l^{D} \delta_{\min }^{2-D}
$$

where $D$ is the complexity of the ubiquitiform and, according to Ou et al. (2014), the value of $D$ for the ubiquitiform is equal to the fractal dimension of its associated fractal. $\delta_{\min }$ is the lower bound to scale invariance for the ubiquitiform, which is believed to be related to the microstructure of the object under consideration.

Substituting Eq. (2.3) into Eq. (2.1), the ubiquitiformal fracture energy $G_{u f}$ of a material can be defined as

$$
G_{u f}=\frac{W}{l^{D}} \delta_{\min }^{2-D}
$$

Moreover, the relationship between the ubiquitiformal fracture energy and the traditional one can be obtained directly from Eqs. (2.4) and (2.2), as

$$
\frac{G_{u f}}{G}=\left(\frac{l}{\delta_{\min }}\right)^{2-D}
$$

It is seen from Eq. (2.5) that, unlike the fractal fracture energy (Borodich, 1992, 1997, 1999; Bažant, 1995, 1997a,b; Carpinteri, 1994; Carpinteri and Ferro, 1994; Carpinteri et al., 2002), the ubiquitiformal fracture energy $G_{u f}$ can be obtained directly from physical and geometrical properties of the object under consideration, and then such a ubiquitiformal fracture energy can be taken as a reasonable material parameter, as was proposed by Addison (2000). However, on the one hand, Addison (2000) reached this conclusion via the concept of a pre-fractal, which implied that the fracture surface was of fractal, i.e. the fracture surface had fractional dimension. On the contrary, the concept of the ubiquitiform emphasis the integral dimension feature of a real object in nature, that is, all the real fracture surfaces are of integral dimension 2 . On the other hand, the pre-fractal fracture energy has to be determined via an ambiguous parameter, namely, the hypervolume $V^{*}$, which is unnecessary in the determination of the ubiquitiformal fracture energy.

Furthermore, it is believed that there is a size effect for the traditional fracture energy $G$, which can be easily obtained from Eq. (2.5). Considering two specimens in different sizes $l_{1}$ and $l_{2}$, respectively, there are

$$
\frac{G_{u f}}{G_{1}}=\left(\frac{l_{1}}{\delta_{\min }}\right)^{2-D} \quad \frac{G_{u f}}{G_{2}}=\left(\frac{l_{2}}{\delta_{\min }}\right)^{2-D}
$$

where $G_{1}$ and $G_{2}$ are the corresponding traditional fracture energies for the two specimens, respectively. And then the size effect can be presented simply as

$$
\frac{G_{1}}{G_{2}}=\left(\frac{l_{1}}{l_{2}}\right)^{D-2}
$$

In fact, Carpinteri and Ferro (1994), Carpinteri and Chiaia (1995) also obtained such a relationship from fractal theory based on the concept of renormalized fracture energy. However, similarly to the concept of the hypervolume used by Addison (2000), the renormalization fracture energy has also no clear physical meaning, and it is difficult to be determined in practice.

\section{Numerical results and discussions}

In Eq. (2.5), both the traditional fracture energy $G$ and the complexity $D$ can be determined experimentally. For concrete material, the lower bound to scale invariance $\delta_{\min }$ can be empirically related to the tensile strength $f_{t}(\mathrm{Li}, 2014)$ as 


$$
\delta_{\min }=221.28 f_{t}^{-3.24}
$$

where the units of $\delta_{\min }$ and $f_{t}$ are $\mu \mathrm{m}$ and $\mathrm{MPa}$, respectively.

To investigate numerically the properties of the ubiquitiform fracture energy, concrete materials presented in Swartz and Kan (1992) as well as by Saouma et al. (1990, 1991) and Saouma and Barton (1994) are used. The corresponding material properties as well as the calculated values of $\delta_{\min }$ from Eq.(3.1) are listed in Tables 1 and 2, respectively. For convenience, according to Addison (2000), the complexities used for the concrete materials presented by Swartz and Kan (1992) are all taken to be $D=2.1$. In the tables, $E$ is the elastic modulus and $K_{I C}$ is the fracture toughness.

Table 1. Experimental data (Swartz and Kan, 1992) and the corresponding lower bound to scale invariance

\begin{tabular}{|c|c|c|c|c|c|c|}
\hline Specimen & $\begin{array}{c}l \\
{[\mathrm{~cm}]}\end{array}$ & $\begin{array}{c}E \\
{[\mathrm{GPa}]}\end{array}$ & $\begin{array}{c}K_{I C} \\
{[\mathrm{MPa} \sqrt{\mathrm{m}}]}\end{array}$ & $\begin{array}{c}G \\
{[\mathrm{~N} / \mathrm{m}]}\end{array}$ & $\begin{array}{c}f_{t} \\
{[\mathrm{MPa}]}\end{array}$ & $\begin{array}{c}\delta_{\min } \\
{[\mu \mathrm{m}]}\end{array}$ \\
\hline \hline NC-.64 & 12.7 & 31.0 & 1.015 & 99.0 & 5.1 & 1.1 \\
\hline HC-.64 & 12.7 & 35.0 & 1.327 & 144.4 & 6.0 & 0.7 \\
\hline NP-.64 & 12.7 & 32.7 & 1.078 & 99.9 & 5.4 & 1.0 \\
\hline NP-.30 & 12.7 & 37.2 & 1.392 & 127.4 & 8.0 & 0.3 \\
\hline HC-.30 & 12.7 & 38.2 & 1.676 & 166.8 & 8.0 & 0.3 \\
\hline NC-.30 & 12.7 & 41.6 & 1.439 & 119.0 & 8.4 & 0.2 \\
\hline
\end{tabular}

Table 2. Experimental data (Saouma et al., 1990, 1991; Saouma and Barton, 1994) and the corresponding lower bound to scale invariance

\begin{tabular}{|c|c|c|c|c|c|c|c|}
\hline Specimen & $\begin{array}{c}l \\
{[\mathrm{~cm}]}\end{array}$ & $\begin{array}{c}E \\
{[\mathrm{GPa}]}\end{array}$ & $\begin{array}{c}D \\
{[-]}\end{array}$ & $\begin{array}{c}K_{I C} \\
{[\mathrm{MPa} \sqrt{\mathrm{m}}]}\end{array}$ & $\begin{array}{c}G \\
{[\mathrm{~N} / \mathrm{m}]}\end{array}$ & $\begin{array}{c}f_{t} \\
{[\mathrm{MPa}]}\end{array}$ & $\begin{array}{c}\delta_{\min } \\
{[\mu \mathrm{m}]}\end{array}$ \\
\hline \hline S32A & 40.64 & 16.9 & 2.1 & 0.89 & 224.6 & 2.67 & 9.2 \\
\hline S32B & 40.64 & 16.9 & 2.098 & 1.0 & 205.3 & 2.67 & 9.2 \\
\hline S32C & 40.64 & 16.9 & 2.117 & 1.1 & 238.6 & 2.67 & 9.2 \\
\hline S52A & 67.74 & 16.9 & 2.073 & 1.16 & 205.3 & 2.67 & 9.2 \\
\hline SS32A & 40.64 & 23.2 & 2.08 & 1.4 & 303.5 & 3.96 & 2.6 \\
\hline SS32B & 40.64 & 23.2 & 2.085 & 1.25 & 249.1 & 3.96 & 2.6 \\
\hline S33A & 40.64 & 16.5 & 2.097 & 0.99 & 212.3 & 2.41 & 12.8 \\
\hline S33B & 40.64 & 16.5 & 2.109 & 0.88 & 221.1 & 2.41 & 12.8 \\
\hline S33C & 40.64 & 16.5 & 2.103 & 1.28 & 245.6 & 2.41 & 12.8 \\
\hline S53A & 67.74 & 16.5 & 2.082 & 0.98 & 236.8 & 2.41 & 12.8 \\
\hline
\end{tabular}

The numerical results of the ubiquitiformal fracture energy calculated by using Eq. (2.5) for the two materials are presented in Figs. 1a and 1b, respectively. For the sake of comparison and discussions, the numerical results of both the traditional fracture energy $G$ and the critical strain energy release rate $G_{c}$ calculated from the fracture toughness relation $G_{c}=K_{I C}^{2} / E$ are also presented in Figs. 1a and 1b.

It can be seen from both Figs. 1a and $1 \mathrm{~b}$ that the numerical result of the ubiquitiformal fracture energy $G_{u f}$ is in good agreement with that of the critical strain energy release rate $G_{c}$ calculated from the fracture toughness relation and, as usuall, far from that of the fracture energy $G$. As is well known, the discrepancy between the calculated results of the critical strain energy release rate $G_{c}$ from the fracture toughness $K_{I C}$ and the experimental data of $G$ has been perplexing researchers for a long time. It was conjectured that, in general, the assumption of linear elasticity is not a so good approximation to describe physical properties of real 
(a)

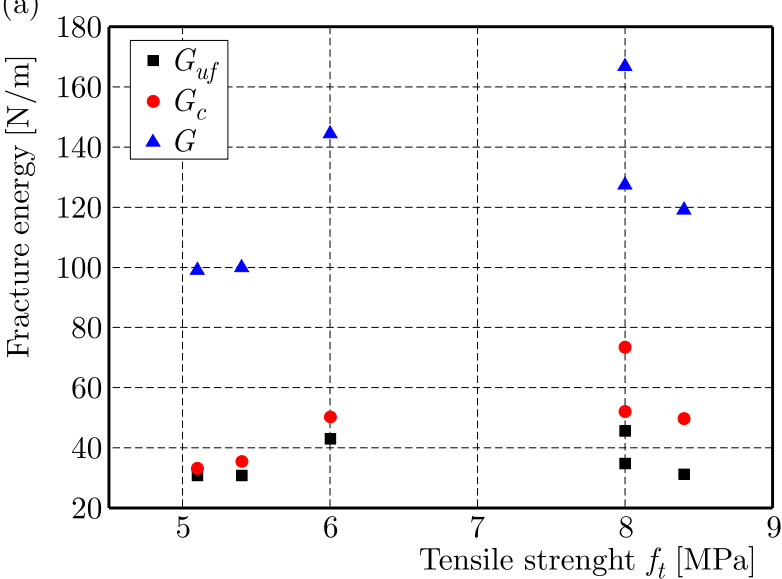

(b)

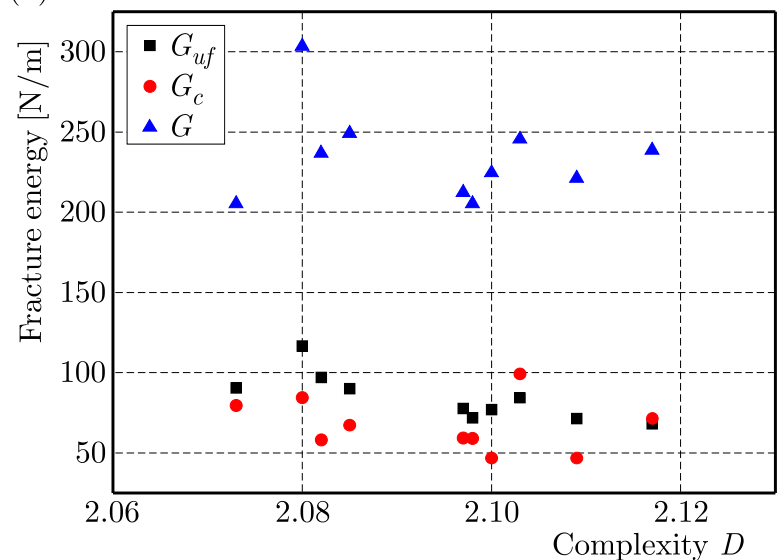

Fig. 1. Ubiquitiformal fracture energy $G_{u f}$ for concrete: (a) Swartz and Kan (1992), (b) Saouma et al. $(1990,1991)$, Saouma and Barton (1994)

materials, because the relation for fracture toughness is derived from rationalistic deduction on the assumption of linear elasticity. It seems now that the above mentioned discrepancy can be reasonably explained based on the ubiquitiformal fracture energy as follows. In fact, in the proper sense, the fracture energy $G$ is obtained by the ratio of the work done by the external traction to the area of the fracture surface, which hence represents the average energy release rate for creating the fracture surface and characterizes a global fracture property of the material under consideration. On the other hand, however, the critical strain energy release rate $G_{c}$ is the critical crack-tip energy release rate, which describes the local fracture property of materials. Thus, it can be realized that the discrepancy between the values of the fracture energy $G$ and the critical strain energy release rate $G_{C}$ should result from two uncertainties coming from the two physical variables $G$ and $G_{c}$, respectively. One is the accuracy of the calculation results of the area of the fracture surface for the fracture energy $G$, and the other one is the availability of the assumption of linear elasticity for deduction of the critical strain energy release rate $G_{c}$ as inferred in the past. Obviously, based on large volumes of the experimental variations as above mentioned, the real fracture surface should be ubiquitiformal rather than smooth. Therefore, it must be questionable to calculate the area of the fracture surface under the smooth surface assumption, and instead of which, the concept of the ubiquitiform surface must be taken into account. Considering further the agreement of the calculated numerical results of the ubiquitiformal fracture energy $G_{u f}$ and that of the critical strain energy release rate $G_{c}$, it can be believed that the above mentioned discrepancy is indeed resulted from the incorrect calculation of the area of the fracture surface on the smooth surface assumption and that the ubiquitiformal fracture energy $G_{u f}$ is superior to the traditional fracture energy $G$. Moreover, it should be pointed out here that the fact that the numerical result of the ubiquitiformal fracture energy $G_{u f}$ is in good agreement with that of the critical strain energy release rate $G_{c}$ calculated from the fracture toughness relation also implies that the opened area of the fracture surface is more important than the well-known crack-tip stress singularity in the description of the fracture process in materials. This can be demonstrated further as follows. On the one hand, $G_{c}$ comes from the rigorous theoretical analysis of the crack-tip stress singularity on the assumption of linear elasticity. On the other hand, a ubiquitiformal crack will include a number of smaller cracks distributed in different lengths and directions, which obviously may result in much more complexity in the crack-tip stress singularity. However, although it does not take the complicated crack-tip stress singularity into account, the numerical results of the ubiquitiformal fracture energy calculated directly via the area of the ubiquitiformal fracture surface can still be in good agreement with that of the critical strain energy release rate, which just verifies the importance of the area. 
Nay more, unlike the fracture energy $G$, for some concrete materials, the ubiquitiformal fracture energy $G_{u f}$ seems to have not the size effect, which is shown in Table 3 by taking the concrete presented by Carpinteri and Ferro (1994) as an example. The material properties as well as the calculated values of both $\delta_{\min }$ and $G_{u f}$ are listed in Table 3, where $G_{m}$ and $G_{u f m}$ are the mean values of the fracture energy $G$ and the ubiquitiformal fracture energy $G_{u f}$, respectively; $E_{r}(G)$ and $E_{r}\left(G_{u f}\right)$ are the relative errors of $G$ and $G_{u f}$, respectively. It can be seen that the relative errors of the ubiquitiformal fracture energy $E_{r}\left(G_{u f}\right)$ are all within the range of $10 \%$ for varying sizes of the specimens, while that of the traditional fracture energy $E_{r}(G)$ can reach up to $30 \%$.

Table 3. Experimental data (Carpinteri and Ferro, 1994) and the corresponding ubiquitiformal fracture energy

\begin{tabular}{|c|c|c|c|c|c|c|c|c|c|}
\hline $\begin{array}{c}l \\
{[\mathrm{~cm}]}\end{array}$ & $\begin{array}{c}f_{t} \\
{[\mathrm{MPa}]}\end{array}$ & $\begin{array}{c}\delta_{\min } \\
{[\mu \mathrm{m}]}\end{array}$ & $\begin{array}{c}D \\
{[-]}\end{array}$ & $\begin{array}{c}G \\
{[\mathrm{~N} / \mathrm{m}]}\end{array}$ & $\begin{array}{c}G_{m} \\
{[\mathrm{~N} / \mathrm{m}]}\end{array}$ & $\begin{array}{c}E_{r}(G) \\
{[\%]}\end{array}$ & $\begin{array}{c}G_{u f} \\
{[\mathrm{~N} / \mathrm{m}]}\end{array}$ & $\begin{array}{c}G_{u f m} \\
{[\mathrm{~N} / \mathrm{m}]}\end{array}$ & $\begin{array}{c}E_{r}\left(G_{u f}\right) \\
{[\%]}\end{array}$ \\
\hline \hline 5 & 4.25 & 2.04 & 2.38 & 83 & 109 & -31 & 1.78 & 1.97 & -10 \\
\hline 10 & 3.78 & 2.98 & 2.38 & 102 & 109 & -7 & 1.94 & 1.97 & -2 \\
\hline 20 & 3.64 & 3.37 & 2.38 & 142 & 109 & 23 & 2.18 & 1.97 & 10 \\
\hline
\end{tabular}

In addition, it can be seen from Eq. (2.5) that the lower bound to scale invariance $\delta_{\min }$ can introduce some errors to the calculation of the area of the ubiquitiformal fracture surface $A_{u f}$ and then affect the calculation results of the ubiquitiformal fracture energy $G_{u f}$. In the following, it will be numerically demonstrated that such an influence can be neglected. Denote the true value and the actual value of the lower bound to scale invariance by $\delta_{\min }$ and $\delta_{\min }^{\prime}$, respectively, the corresponding areas of the ubiquitiformal fracture surface by $A_{u f}$ and $A_{u f}^{\prime}$, and the relative error of $\delta_{\text {min }}$ and of the ubiquitiformal area $A_{u f}$ by $E_{r}\left(\delta_{m i n}\right)$ and $E_{r}\left(A_{u f}\right)$, respectively, one can obtain the relation between the two relative errors from Eq. (2.3), as

$$
\begin{aligned}
& E_{r}\left(\delta_{\text {min }}\right)=\frac{\delta_{\text {min }}^{\prime}-\delta_{\min }}{\delta_{\min }}=\frac{\delta_{\min }^{\prime}}{\delta_{\min }}-1 \\
& E_{r}\left(A_{u f}\right)=\frac{A_{u f}^{\prime}-A_{u f}}{A_{u f}}=\left(\frac{\delta_{\min }^{\prime}}{\delta_{\min }}\right)^{2-D}-1=\left[E_{r}\left(\delta_{\min }\right)+1\right]^{2-D}-1
\end{aligned}
$$

Thus, from Eqs. (3.2), the relative error $E_{r}\left(A_{u f}\right)$ only depends on the relative error $E_{r}\left(\delta_{\min }\right)$ and the complexity $D$. For example, for $D=2.1$, taking a larger value of the relative error of the lower bound to scale invariance $E_{r}\left(\delta_{\min }\right)=50 \%$, it can be calculated from Eqs. (3.2) that $E_{r}\left(A_{u f}\right)=-3.97 \%$, which is obviously an acceptable error in most engineering applications.

\section{Conclusion}

Based on the new concept of ubiquitiform, namely, all the real physical or geometrical objects in nature are ubiquitiformal, the fracture energy, one of the important mechanical properties in the fracture mechanics, is re-examined in this study. Instead of the traditional fracture energy $G$ for the smooth crack configuration, the concept of the ubiquitiformal fracture energy $G_{u f}$ is proposed. Because of the integral dimension characteristic of a ubiquitiform crack or the corresponding ubiquitiform fracture surface, an explicit expression for the ubiquitiformal fracture energy can be obtained, which is intrinsically different from the case for a fractal crack because of the singularity of the integral dimension of fractals. Moreover, it is found that the calculated numerical results of the ubiquitiformal fracture energy are in good agreement with those for the critical strain energy release rate $G_{c}$ calculated from the fracture toughness relation, $G_{c}=K_{I C}^{2} / E$. Consequently, the perplexity over a long period of time about the discrepancy 
between the experimental data of the traditional fracture energy $G$ and the calculated results of the critical strain energy release rate $G_{c}$ by using the fracture toughness relation can be reasonably explained. That is, the fracture surfaces generated in a real material cannot be thought of as a smooth configuration but, instead, it must be a ubiquitiformal one, and then, instead of the traditional fracture energy, the ubiquitiformal fracture energy must be adopted in practical engineering applications. In addition, it should be pointed out that the agreement between the numerical results of $G_{u f}$ and $G_{c}$ also implies that the created area of the fracture surface will play a more important role than the crack-tip stress singularity to characterize the fracture process in materials. Finally, unlike the traditional fracture energy, for some concrete materials, it is verified numerically that there is not size effect for the ubiquitiformal fracture energy, which, certainly, should be further theoretically studied in future.

\section{Acknowledgements}

The project has been supported by both the National Natural Science Foundation of China under Grant 11221202 and the National Science Foundation of China under Grant 11390362.

\section{References}

1. Addison P.S., 2000, The geometry of prefractal renormalization: Application to crack surface energies, Fractals, 8, 2, 147-153

2. BAŽAnt Z.P., 1995, Scaling of quasi-brittle fracture and the fractal question, ASME Journal of Materials and Technology, 117, 361-367

3. BAŽAnt Z.P., 1997a, Scaling in nonlinear fracture mechanics, IUTAM Symposium on Nonlinear Analysis of Fracture, University of Cambridge, 3-7 September 1995, J.R. Willis (Edit.), Kluwer Academic Publishers, Dordrecht, 1-12

4. BAŽANT Z.P., 1997b, Scaling of quasibrittle fracture: hypotheses of invasive and lacunar fractality, their critique and Weibull connection, International Journal of Fracture, 29, 1699-1709

5. BAŽANT Z.P., YAVARI A., 2005, Is the cause of size effect on structural strength fractal or energeticstatistical?, Engineering Fracture Mechanics, 72, 1-31

6. Borodich F.M., 1992, Fracture energy in a fractal crack propagating in concrete or rock, Doklady Rossiyskoy Akademii Nauk, 325, 6, 1138-1141

7. Borodich F.M., 1997, Some fractal models of fracture, Journal of the Mechanics and Physics of Solids, 45, 2, 239-259

8. Borodich F.M., 1999, Fractals and fractal scaling in fracture mechanics, International Journal of Fracture, 95, 1-4, 239-259

9. CARPinteri A., 1994, Fractal nature of material microstructure and size effects on apparent mechanical properties, Mechanics of Materials, 18, 2, 89-101

10. Carpinteri A., Chiaia B., 1995, Multifractal nature of concrete fracture surfaces and size effects on nominal fracture energy, Materials and Structures, 28, 435-443

11. Carpinteri A., Ferro G., 1994, Size effects on tensile fracture properties: a unified explanation based on disorder and fractality of concrete microstructure, Materials and Structures, 27, 563-571

12. CARPinteri A., Puzzi S., 2008, Self-similarity in concrete fracture: size-scale effects and transition between different collapse mechanisms, International Journal of Fracture, 154, 1-2, 167-175

13. Krohn C.E., Thompson A.H., 1986, Fractal sandstone pores: Automated measurements using scanning-electron-microscope images, Physical Review B, 33, 6366-6374

14. LI G.Y., 2014, Research on basic theory of ubiquitiform and some aspects of its application (in Chinese), PhD Thesis, Beijing Institute of Technology, Beijing 
15. Mandelbrot B.B., Passoja D.E., Paullay A.J., 1984, Fractal character of fracture surfaces of metals, Nature, 308, 721-722

16. Mecholsky J.J., Passoja D.E., Feinberg-Ringle K.S., 1989, Quantitative analysis of brittle fracture surfaces using fractal geometry, Journal of the American Ceramic Society, 72, 60-65

17. Ou Z.-C., Li G.-Y., DuAn Z.-P., HuAng F.-L., 2014, Ubiquitiform in applied mechanics, Journal of Theoretical and Applied Mechanics, 52 , 1, 37-46

18. Radliński A.P., Radlińska E.Z., Agamalian M., Wignall G.D., Lindner P., Randl O.G., 1999, Fractal geometry of rocks, Physical Review Letters, 31, 163-172

19. Saouma V.E., Barton C.C., 1994, Fractals, fractures, and size effects in concrete, Journal of Engineering Mechanics, 120, 4, 835-854

20. Saouma V.E., Barton C.C., Gamaleldin N.A., 1990, Fractal characterization of fracture surfaces in concrete, Engineering Fracture Mechanics, 35, 1-3, 47-53

21. Saouma V.E., Broz J.J., Brühwiler E., Boggs H.L., 1991, Effect of aggregate and specimen size on fracture properties of dam concrete, Journal of Materials in Civil Engineering ASCE, 3, 3, 204-218

22. SwArTZ S., KAn Y.C., 1992, The influence of aggregate/paste bonding and strength of mode I fracture mechanics properties of concrete, [In:] Fracture Mechanics of Concrete Structures, Bažant Z.P. (Edit.), Elsevier Applied Science, London, 437-442

23. Underwood E.E., 1986, Fractals in fractography, Materials Science and Engineering A, 80, 1-14 